Documentación de las Ciencias de la Información ISSN-e: $1988-2890$

https://dx.doi.org/10.5209/dcin. 77478

\title{
SEO para periodistas: nuevo perfil profesional y estrategias de publicación desde la redacción
}

\author{
Magdalena Trillo Domínguez; Celia Gallego Márquez²
}

Recibido: 29 de julio de 2021 / Aceptado: 2 de octubre de 2021

Resumen. Con la misma fuerza con que los buscadores compiten con los medios como intermediarios en el acceso a la información, la cultura SEO empieza a penetrar en las redacciones poniendo de manifiesto que ya no es suficiente con incorporar expertos en optimización de contenidos (ni siquiera la creación de un departamento especializado). El impacto de los algoritmos está transformando el perfil del periodista digital, obligándole a aprender habilidades de producción, edición y publicación SEO, y modificando la propia agenda setting de los medios incorporando las tendencias y temas de interés de las audiencias. En este trabajo se realiza un acercamiento a las técnicas de posicionamiento en buscadores con el objetivo de explorar, desde la praxis del ejercicio del oficio en el corazón de las redacciones, las oportunidades que supone este perfil emergente tanto a nivel profesional como académico para estudiantes de Ciencias de la Información y la Comunicación. Se realiza en un sentido constructivo, la viabilidad misma de las empresas periodísticas depende en buena medida de la pata de tráfico que llega del SEO, pero también crítico alertando de las consecuencias que estas pautas de trabajo pueden suponer en el ejercicio del periodismo. Justo en un momento de desinformación y saturación como el actual, el papel de intermediarios de los medios se presenta más necesario que nunca y, paradójicamente, también está más competido que nunca por los algoritmos.

Palabras clave: SEO, periodismo, posicionamiento web, algoritmos, buscadores, redacción

\section{[en] SEO for journalists: new professional profile and publication strategies from de newsroom}

\begin{abstract}
The SEO culture begins to penetrate newsrooms, showing that it is no longer enough to incorporate experts in content optimization (not even the creation of specialized departments) and it is necessary going one step forward. The impact of the algorithms is transforming the profile of the digital journalists, forcing them to learn SEO production, editing and publication skills, and modifying the media's own agenda setting, giving trends and topics of interest to audiences. In this article, an approach is made to Search Engine Optimization techniques with the aim of exploring, from journalism practice in the core of newsrooms, the opportunities that this emerging profile represents professionally and academically. This work is carried out in a constructive sense, the viability of journalistic companies depends largely on the traffic branch that comes from SEO, but it is also a critical warning of the consequences that these work guidelines can entail in the exercise of Journalism. In this time of disinformation and saturation, the role of media intermediaries appears more necessary than ever and, paradoxically, it is also more disputed than ever by algorithms.
\end{abstract}

Keywords: SEO, journalism, search engine optimization, algorithms, newsroom

Sumario. 1. Introducción 2. Marco teórico 3. Método y objetivos 4. Resultados y recomendaciones. 5. Conclusiones y discusión 6. Referencias bibliográficas.

Cómo citar: Trillo Domínguez, M.; Gallego Márquez, C. (2022): SEO para periodistas: nuevo perfil profesional y estrategias de publicación desde la redacción, en Documentación de Ciencias de la Información 45 (1), 17-24.

\section{Introducción}

La expansión social y profesional de internet y las redes sociales han provocado un cambio de paradigma en los procesos de comunicación que está afectando de lleno al oficio periodístico: desde la nueva relación que se establece entre los profesionales de la información y las audiencias hasta las estrategias que se están imponiendo en las redacciones en cuanto a lenguaje, narrativas y formatos (Sa- laverría, 2005; Trillo \& Alberich, 2020) pero también respecto a las pautas de publicación orientadas a garantizar la visibilidad de los contenidos en el competitivo tablero digital. Los periodistas escriben para ser leídos (Giomelakis, Karypidou \& Veglis, 2019) y para que sus artículos sean encontrados: si el público cada vez busca más los contenidos a través de buscadores, con Google en una situación de casi monopolio, no hay más recorrido que aprender a posicionar las historias incorporando las nuevas

\footnotetext{
Universidad de Granada / Diario de Sevilla (Grupo Joly).

E-mail: mtrillo@ugr.es

ORCID: 0000-0001-9505-1203

2 Universidad de Granada

E-mail: celiagm@correo.ugr.es

ORCID: https://orcid.org/0000-0001-6706-1762
} 
reglas del juego (Lopezosa, Codina \& Pérez-Montoro, 2019).

En síntesis, estas ideas de base son las que tienen que ver con el pujante Periodismo SEO y las que han sostenido buena parte de los estudios e investigaciones que se ha realizado tanto en el terreno académico como profesional en torno a dos evidencias: que hace falta una formación SEO reglada y sólida (Lopezosa et al., 2020) que dé cobertura a los perfiles emergentes que están despuntando en este campo y que se presenta como una cuestión de oportunidad y hasta de supervivencia para las empresas periodísticas competir con los grandes buscadores y agregadores de información posicionando sus contenidos.

El llamado tráfico orgánico, el que de forma natural consiguen los sites a través de los motores de búsquedas, ocupa un lugar cada vez más determinante en la posición relativa de los medios en los nuevos ranking de audiencia digital (Del Castillo, 2021): es una consecuencia de los nuevos hábitos de lectura y consumo de información y resulta vital para los medios por lo que significa para su propia posición de marca y por su repercusión en los ingresos a través de la publicidad.

Estamos, además, ante una tendencia en continuo crecimiento como revela el último Digital News Report (Amoedo, 2021): seis de cada diez internautas (57\%) utilizan ya una vía algorítmica para acceder a la información, ya sea un agregador de contenidos, las redes sociales o la búsqueda de noticias concretas. Según la encuesta de 2021, la mitad de los usuarios acude directamente a la marca del medio a través del sitio web o aplicación del mismo, pero también buscando el site a través del buscador.

En este contexto de disrupción profesional, la cultura SEO empieza a penetrar en las redacciones de los medios poniendo de manifiesto que ya no es suficiente con incorporar un experto en SEO y ni siquiera creando un departamento especializado en la generación de este tipo de contenidos. Está ocurriendo como ya pasó hace más de dos décadas, en los albores de los cibermedios, cuando se dio el salto digital: primero se encapsuló la apuesta a la espera de ver si internet era una moda hasta que se asumió que el desafío era tal que no había más camino que afrontar la transformación digital de una forma integral.

La apuesta SEO se empieza a generalizar en estos momentos siguiendo un esquema similar: son necesarios los perfiles y equipo especializado (sobre todo técnico) pero es preciso extender unos conocimientos mínimos de forma generalizada a toda la redacción. Porque las noticias vuelan solas en la web, pero llegan más lejos si se les ayuda.

El desarrollo del SEO desde dentro de las redacciones, con el impacto que está significando para el día a día del periodista, es el eje del presente trabajo, una investigación que se construye desde abajo explorando las rutinas y las estrategias de búsqueda de contenidos, edición y publicación que están marcando la nueva agenda de los medios.

\section{Marco teórico}

En el nuevo ecosistema digital caracterizado por la inmediatez, la globalidad y la sobreinformación, también el mundo del Periodismo ha de adaptarse a los nuevos tiempos marcados por los avances tecnológicos con internet como herramienta de acceso y distribución masiva de información. El reto supone pasar al periodismo 3.0, lo que acarrea una transformación en las estructuras de la profesión y en las formas de tratar el proceso de comunicación con un papel de las audiencias cada vez más determinante: han pasado de ser simples receptores a tener unas capacidades de emisión e implicación en los procesos que ha determinado tanto el Periodismo Ciudadano (Sánchez-Guijaldo, 2017) como el Periodismo Participativo (Cabezuelo \& Escanciano, 2007) en "una conversación de muchos a muchos" donde "el contenido y el criterio de los usuarios y de la comunidad se convierte en la guía y principal elemento de jerarquización de contenidos" (Varela, 2005: 68-76).

El desarrollo de los medios en la Red y el planteamiento del periodismo 3.0 son inseparables de la expansión de la Web 2.0, que da soporte a una sociedad de información, comunicación y conocimiento donde son clave los nuevos paradigmas de la e-comunicación (Orihuela, 2004) con el paso del concepto de audiencia a la de usuario, el auge de los contenidos multimedia, la dictadura del tiempo real y el paso de lo lineal a lo interactivo e hipertextual propiciando la "desintermediación" que marca el nuevo ecosistema y que da pie, justamente, a que motores de búsqueda, metabuscadores, índices temáticos, portales y páginas de recursos intervengan ayudando (y compitiendo con los medios) en la búsqueda de información y acceso a todo tipo de contenidos.

Ya no hablamos de productos cerrados sino de servicios y de un entorno fluido en que los papeles de emisor y receptor no solo intercambian, también se ven afectados por la acción de los buscadores que son los que ahora cogen el testigo de los medios haciendo de intermediarios entre los contenidos periodísticos y los usuarios. En este nuevo entorno de conexión, es fundamental atender a la "visibilidad web" en la medida en que, de todos los resultados que los algoritmos recuperarán para el usuario, los que aparezcan primero tendrán más posibilidades de ser leídos.

Junto con los buscadores, también las redes sociales ocupan una posición estratégica en el nuevo paradigma de comunicación (Caldevilla, 2010; Parra \& Onieva, 2020) y también aquí entran en juego los algoritmos: las plataformas actúan como altavoz para propagar el contenido emitido entre una audiencia mucho más amplia que además reacciona ante él, compartiéndolo, valorándolo, reflexionando o incluso ampliándolo para conseguir más interacciones y engagement (Curiel, 2011).

Cómo lograr que una historia llegue al lector, que sea visible y resulte lo suficientemente interesante y atractiva para que decida leerla... En este plantea- 
miento de síntesis podríamos decir que se sitúa el origen del perfil del periodista SEO. En realidad, buena parte del desarrollo que los medios están realizando se orienta a conseguir nuevas audiencias y está suponiendo una modificación de la teoría de la Agenda Setting (McCombs y Shaw, 1972).

Es decir, los criterios profesionales por los que los periodistas deciden a diario qué es noticia han cambiado y están determinando incluso una nueva reorganización de las redacciones (de las tradicionales secciones a equipos de producción de contenidos donde un pilar estratégico es, justamente, dar respuesta a las "tendencias" de búsqueda y conversación desde el periodismo de servicios).

Ahora, gracias a estos nuevos desarrollos tecnológicos y el hecho de que podemos observar y monitorizar qué está buscando la gente, los periodistas pueden decidir el establecimiento de una parrilla de contenidos adaptada a las necesidades de los lectores. Aunque no siempre la labor profesional tiene por qué coincidir con los datos que proporciona el SEO (el criterio editorial se mantiene), es evidente que éste ha cambiado la forma de trabajar de los medios, pues indica otra manera de ver cuáles son las necesidades de las audiencias y puede suponer, incluso, una guía para el periodista.

Partimos así de la "agenda de los medios" (lo que no entra ahí, no existe) y de la figura del gatekeeper (el periodista como guardián o portero de la actualidad) como ideas centrales de una etapa de oro de los mass media que también podemos utilizar como símbolo de ese cambio del paradigma que ha impuesto la sociedad red y que afecta a las propias rutinas periodísticas en el día a día de la redacción.

En este escenario, y al margen de desarrollos evolutivos y mejoras técnicas, hoy día los motores de búsqueda se consideran como uno de los servicios más fiables y compartidos para recuperar información en internet y el principal recurso utilizado por millones de usuarios a nivel mundial (Dod, 2017; Purcell, 2011). Asimismo, estudios recientes destacan la tendencia creciente a utilizarlos para sus compras online y la recopilación de información relevante, incluso por encima de las redes sociales y entre el público millennial (Murga, 2017), y corroboran que se han convertido en una de las mejores fuentes de tráfico para cualquier web (Schwartz, 2018). También ha de tenerse en cuenta que buena parte del tráfico de la búsqueda procede de los resultados situados en los primeros puestos (Brightedge, 2018).

Es en este contexto de dinámicas donde se abre camino el SEO referido a las estrategias de posicionamiento y optimización; como conjunto de acciones orientadas a mejorar el posicionamiento de un sitio web en la lista de resultados de los buscadores. Es decir, permite realizar el proceso de mejora de la visibilidad en los resultados orgánicos (el que consi- gue una web de forma espontánea, sin que medie una campaña publicitaria de pago).

Otro motivo clave de la creciente importancia del SEO es que permite que el sitio web sea útil, tanto para los usuarios como para los motores de búsqueda, al tiempo que resulta fundamental para las actuales vías de financiación de los cibermedios (especialmente en lo referente a la publicidad programática). A pesar de que los buscadores no posean razón crítica humana, el SEO les ayuda a comprender acerca del contenido de cada página web y si resulta útil para el usuario situando, por tanto, dicho contenido en una posición relevante que hará que llegue a un número mayor de receptores, aumentando su tráfico y beneficios (Iglesias-García \& Codina, 2016).

Como resultado del impacto tecnológico, también en los últimos años el SEO ha ido evolucionando como reflejan todos los desarrollos recientes en torno a la inteligencia artificial y las búsquedas por voz (Sentance, 2016), una línea de investigación que tiene un reflejo directo en el entorno mediático por la oportunidad de posicionamiento que supone en los buscadores y por el recorrido que todo lo audiovisual (Durán \& Caldera, 2014) implica en los nuevos formatos.

\section{Método y objetivos:}

Investigar el impacto del SEO en el Periodismo desde dentro de las redacciones, a partir de la transformación que se está produciendo en las rutinas de producción, edición y publicación de los profesionales, se conforma como eje de la presente investigación que se articula en torno a una metodología triangular:

En primer lugar, se aborda la revisión sistemática del emergente campo del SEO con una perspectiva académica y de investigación, pero también desde el terreno profesional por su vinculación con las nuevas vías de financiación y viabilidad misma de las empresas periodísticas y por la repercusión que está suponiendo tanto en la transformación de la agenda setting de los medios (Arugete, 2009) como en el perfil del periodista. Para la revisión sistemática se sigue la metodología desarrollada por autores clave en este campo como Codina (2020), Booth, Papaionnou y Sutton (2012) y Hart (2008) en cuanto al proceso de búsqueda, evaluación, análisis y síntesis y al planteamiento del trabajo siguiendo la estructura de IMRyD. Se combinan las fuentes académicas especializadas más relevantes del área (Scopus, Web of Science y Google Scholar) con los informes profesionales del sector, ya que se trata de una investigación de mayor recorrido, tanto en el punto de partida como en su continuación a futuro, al formar parte del trabajo de estudio y especialización de una de las autoras.

En segundo lugar, se lleva a cabo una observación participante de tres meses en Diario de Sevilla ${ }^{3}$, des-

Diario de Sevilla (www.diariodesevilla.es) es una de las nueve cabeceras de Grupo Joly (compañía editora de referencia en Andalucía y decana de 
de el 3 de noviembre de 2020 hasta el 31 de enero de 2021, con incorporación en el equipo Digital y con competencias específicas en el desarrollo de contenidos SEO. La transformación digital, con la implantación de estrategias SEO, se inició en el verano de 2018 y se ha logrado pasar de menos de 3 millones de usuarios únicos a más de 15 en apenas tres años.

El proyecto comenzó con la incorporación de un periodista SEO y un plan de formación, orientación y asesoramiento del equipo técnico de Henneo (con estancias de su responsable en la sede de Sevilla) para las redacciones de los 9 periódicos del grupo. El objetivo del proyecto no era crear un departamento específico sino integrarlo en las rutinas de la redacción como parte de la cultura de funcionamiento diario, si bien hay tanto un responsable SEO como un grupo de redactores del equipo Digital y de Páginas del Sur (empresa coparticipada por Joly para el refuerzo de contenidos) que centran su jornada laboral en el desarrollo de piezas SEO. Es en este equipo donde se realiza la observación participante ${ }^{4}$ como trabajo de campo personalizado, en el marco del método etnográfico, inductivo, dialógico e integral (Angrosino, 2012) como investigación particular en las ciencias sociales.

Completa la investigación la implicación de la entonces responsable del proyecto de Transformación Digital (Magdalena Trillo) con la supervisión diaria del proceso de observación participante (cuyas conclusiones y valoración se recogen en este trabajo), así como del jefe de Audiencias y SEO (Francisco Barquilla) con una entrevista semiestructurada orientada a esclarecer y reforzar los hallazgos y vacíos detectados y que se plantea siguiendo las recomendaciones metodológicas de autores como Vallés (2002). En la entrevista se diferencia el perfil profesional del periodista tradicional y el del periodista SEO, reforzando la importancia del segundo, que es capaz de monitorizar y optimizar el posicionamiento de los resultados de búsqueda mediante una serie de herramientas como Search Console de Google, Semrush o Sistrix, sumando, a su vez, habilidades comunicativas.

Con la investigación se realiza un acercamiento al funcionamiento de las técnicas de posicionamiento en buscadores con el objetivo de explorar las oportunidades de esta nueva línea de trabajo que puede tener utilidad tanto a nivel académico para estudiantes de Ciencias de la Información y Comunicación como a nivel profesional para quienes estén interesados en la especialización SEO. Desde esta perspectiva, se presenta como objetivos centrales del presente trabajo:

- Definir el perfil emergente del periodista SEO en el actual entorno mediático digital desde la práctica del oficio

- Identificar herramientas, pautas, técnicas y estrategias SEO aplicables en los cibermedios para aumentar el tráfico de visitas y conseguir una buena visibilidad web

- Concienciar sobre la importancia de implantar una verdadera cultura SEO, más allá de la incorporación de perfiles especializados, en las redacciones de los actuales medios digitales

\section{Resultados y recomendaciones: periodismo SEO desde dentro}

Las empresas periodísticas, como cualquier otra organización que se desenvuelve en el entorno digital, reconocen la importancia del SEO dentro de las redacciones digitales como estrategia para captar usuarios y en respuesta a la tendencia ya comentada de que cada vez son más los lectores que consumen las noticias mediante los buscadores y no a través del tráfico directo (accediendo directamente sin pasar por los motores de búsqueda).

Se trabajan aspectos técnicos como la optimización de la estructura y los metadatos de una web, pero también se aplica a nivel de contenidos, con el objetivo de volverlos más útiles y relevantes para los usuarios, de ahí a que, aunque existen muchos factores en los que un motor de búsqueda se base para posicionar una página u otra, dos de ello son clave: la autoridad y la relevancia.

A la hora de ejecutar un buen SEO, hay que contar con las herramientas de posicionamiento idóneas. En el ámbito digital, existen numerosos recursos por lo que saber elegir es fundamental con objeto de poder adaptarlo a las características y necesidades de cada sitio web (Luna, 2017). Como síntesis de las más utilizadas y valiosas, aportamos una clasificación de utilidad también en el contexto periodístico:

la prensa española que nació a finales del XIX con la fundación de Diario de Cádiz en 1867 y que se ha expandido a partir de los años 90 por toda la región). En Diario de Sevilla, donde si sitúan los servicios centrales del grupo, trabajan 82 personas y 300 en toda la compañía.

4 La observación participante sigue la experiencia realizada por Carlos Lopezosa en septiembre 2018 justo en el arranque del proyecto de transformación digital e implantación de estrategias SEO. Los resultados y metodología se recogen en este artículo (http://nuevaepoca.revistalatinacs.org/ index.php/revista/article/view/972) 
Tabla 1. Herramientas SEO para periodistas

\begin{tabular}{|c|c|c|}
\hline & $\begin{array}{l}\text { SUITES DE HERRAMIEN- } \\
\text { TAS SEO }\end{array}$ & $\begin{array}{ll}- & \text { Ahrefs } \\
- & \text { Dinorank } \\
- & \text { Semrush } \\
- & \text { SE Ranking } \\
- & \text { Serpstat } \\
- & \text { Mangools SEO Suite } \\
- & \text { Sistrix } \\
- & \text { SEO Powersuite }\end{array}$ \\
\hline $\begin{array}{l}\text { HERRAMIENTAS SEO } \\
\text { GENERALES }\end{array}$ & $\begin{array}{l}\text { HERRAMIENTAS DE SEO } \\
\text { TÉCNICO }\end{array}$ & $\begin{array}{ll}- & \text { Screaming Frog SEO Spider } \\
- & \text { Botify } \\
- & \text { ScrapeBox } \\
- & \text { Bright Local } \\
- & \text { Deep Crawl } \\
- & \text { On Crawl } \\
- & \text { SEO Crawl } \\
- & \text { SEO Site Checkup } \\
- & \text { Siteliner } \\
- & \text { Google Mobile-Friendly } \\
- & \text { Varvy SEO Tool } \\
- & \text { Ahrefs Webmaster Tools } \\
- & \text { Bing Webmaster Tools }\end{array}$ \\
\hline \multirow{8}{*}{$\begin{array}{l}\text { HERRAMIENTAS SEO } \\
\text { ESPECIALIZADAS }\end{array}$} & $\begin{array}{l}\text { CONSTRUIR LISTA DE PA- } \\
\text { LABRAS CLAVE }\end{array}$ & $\begin{array}{ll}- & \text { Google Keyword Planner } \\
- & \text { Keywords Everywhere } \\
- & \text { Surfer SEO } \\
- & \text { Keyword Shitter } \\
- & \text { Google Trends } \\
- & \text { Keyword Tool } \\
- & \text { KWFinder de Mangools } \\
- & \text { ERPChecker de Mangools } \\
- & \text { Answer The Public } \\
- & \text { Also Asked } \\
- & \text { KeywordTool.io } \\
- & \text { Long Tail Pro } \\
- & \text { UberSuggest }\end{array}$ \\
\hline & $\begin{array}{l}\text { MONITORIZAR POSICIÓN } \\
\text { DE PALABRAS CLAVE EN } \\
\text { BUSCADORES }\end{array}$ & $\begin{array}{ll}\text { - } & \text { SERPWatcher } \\
\text { - } & \text { Serpstat Rank Tracker } \\
\text { - } & \text { Ahrefs Rank Tracker } \\
\text { - } & \text { Nightwatch, Advanced Web Ranking } \\
- & \text { TrueRanker } \\
\text { - } & \text { Local Search Results }\end{array}$ \\
\hline & $\begin{array}{l}\text { ANALIZAR EL PERFIL DE } \\
\text { ENLACES }\end{array}$ & \begin{tabular}{|ll}
- & SpyGlass \\
- & Majestic \\
- & LinkMiner de Mangools \\
- & LinkAssistant \\
- & Link Patrol \\
\end{tabular} \\
\hline & $\begin{array}{l}\text { USO DE GESTORES DE } \\
\text { CONTENIDO Y SUS PLU- } \\
\text { GINS ESPECIALIZADOS }\end{array}$ & $\begin{array}{ll}- & \text { Yoast SEO } \\
- & \text { Rank Math SEO } \\
- & \text { SEOPress }\end{array}$ \\
\hline & $\begin{array}{l}\text { ANALIZAR LOS CONTENI- } \\
\text { DOS E INTERACCIONES } \\
\end{array}$ & $\begin{array}{ll}- & \text { Google Analytics } \\
- & \text { Yandex Metrica } \\
\end{array}$ \\
\hline & REALIZAR AUDITORÍAS & $\begin{array}{ll}- & \text { Search Console de Google } \\
- & \text { Bing Webmaster } \\
- & \text { Website Auditor } \\
- & \text { Raven Tools }\end{array}$ \\
\hline & $\begin{array}{l}\text { COMPROBAR LA VELOCI- } \\
\text { DAD DE CARGA }\end{array}$ & $\begin{array}{ll}- & \text { GTmetrix } \\
- & \text { Webpagetest } \\
- & \text { PageSpeed Insights }\end{array}$ \\
\hline & $\begin{array}{l}\text { CONTAR CON UN BUEN } \\
\text { SERVICIO DE HOSTING }\end{array}$ & $\begin{array}{ll}- & \text { Raiola Networks } \\
\text { - } & \text { SiteGround }\end{array}$ \\
\hline
\end{tabular}


A la hora de redactar un contenido online, además de aprovechar las herramientas SEO, se deben tener en cuenta los temas tendencia y elaborar un contenido de calidad a partir de ellos, con una buena estructura, haciendo un buen uso de las imágenes o elementos audiovisuales y siguiendo una serie de pautas que ayudarán a conseguir textos más atractivos para los lectores y para los motores de búsqueda. Desde el día a día del trabajo del periodista SEO en una redacción, aportamos los siguientes:

- Hacer uso de palabras clave correctas y, para ello, se debe pensar qué búsqueda harían los usuarios que podrían estar interesados en el contenido que vamos a tratar en nuestro texto periodístico. Siempre hay que ponerse en el lugar del lector: ¿cómo lo buscaría yo?, ¿qué términos utilizaría? Las keywords se encontrarán en el titular, en la entradilla, en el cuerpo de la noticia, en el cintillo, en los metadatos de las imágenes de las noticias, así como en su descripción o pie de foto (Lopezosa, Trillo \& Codina, 2021). Además, deben estar presentes en el texto de forma natural, su correcto uso dentro de los contenidos hará más visible su información dentro de los resultados de un motor de búsqueda, consiguiendo más tráfico de lectores.

- Elaborar titulares atractivos que capten tráfico de lectores y tengan en cuenta las palabras clave a posicionar (aquellas palabras más consultadas por los usuarios en los buscadores). Es importante incentivar el acceso de los usuarios sin caer en clickbait, por lo que los titulares deben ser siempre fieles al contenido de la cuestión y no dar lugar a expectativas falsas.

- Dividir el texto en párrafos y crear una estructura fácil de seguir que permita distinguir los apartados. Se pueden añadir subtítulos utilizando encabezados $h$ que poseían distintas etiquetas $\mathrm{H} 2$, $\mathrm{H} 3$... logrando un contenido jerarquizado, organizado y más fácil de leer.

- Adjuntar enlaces internos y externos, que promocionen al propio medio favoreciendo además la experiencia del usuario y mejoren la indexación o que añadan información relacionada adicional de fuentes fiables. Del mismo modo, se recomienda la utilización de tags que direccionen a una nueva página que incluirá artículos relacionados con ese tema y mostrará el despliegue de contenidos (la apuesta del medio) por un determinado tema. Dará, por tanto, autoridad al site.

- Añadir imágenes de calidad y libres de derechos de autor. Cualquier noticia debe incluir una imagen atrayente y relacionada, no obstante, la inclusión de más imágenes, vídeos, gifs o elementos audiovisuales en general a la publicación amenizará la lectura. De igual modo, añadir texto adicional en las fotos permitirá posicionarlas en búsquedas por imágenes. Otro factor importante es el atributo ALT de la imagen, ya que informa sobre su contenido y permite al buscador contextualizar la imagen.

- Optimizar la URL, elaborándola con palabras clave y relacionadas con la temática del artículo que se está escribiendo. Todas aquellas palabras que no aporten valor como palabras no relevantes, preposiciones o conjunciones no deben aparecer en la URL.

- Aplicar el formato de negrita, cursiva y subrayado del texto de forma adecuada. La negrita destacará las palabras más importantes, incluyendo palabras clave, de esta forma se le indicará tanto al lector como al buscador lo que se ha considerado relevante. La cursiva se utilizará para títulos, citas o extranjerismos. El subrayado, por su parte, en textos periodísticos no suele emplearse, ya que a menudo provoca confusión con los enlaces, por lo tanto, solo se utilizará en momentos muy específicos.

- Elaborar una conclusión clara y concisa del artículo que incluya algunas de las palabras clave principales siempre será enriquecedor y terminará de ofrecer una estructura atractiva y útil para el lector.

- Compartir en redes sociales y analizar las publicaciones para saber qué contenido se mueve en ellas (de esta forma tenemos controlados a los usuarios y la competencia). Resulta fundamental, en el escenario de horizonte transmedia, adaptar el contenido y recursos a las diferentes redes sociales modificando la redacción periodística, estilo, recursos, etc.

Un buen periodista (digital o no) debe hacer un buen uso del lenguaje, ejecutar una correcta redacción y sintaxis y saber jugar con las palabras para elaborar textos persuasivos que provoquen emociones en el lector. El periodista digital debe dar un paso más allá: tener creatividad y una visión estratégica para que sus contenidos tengan engagement. Esta visión estratégica hace referencia al hecho de que el periodista debe servir a la audiencia, sabiendo responder a sus necesidades, aprovechando los recursos digitales y añadiendo contenido audiovisual, pues el poder de la imagen y el vídeo también atraerán tráfico.

Hablamos así ya del periodista SEO como profesional capaz de elaborar contenido original, de calidad, atractivo y orientado al usuario. En este caso, debe dominar el conocimiento técnico que implica la disciplina SEO, desde aplicar acciones de SEO básicas (titular, imagen y resto del texto optimizado para buscadores) a sus noticias y contenidos periodísticos hasta elaborar informes sobre resultados de audiencias, tendencias de búsqueda e investigaciones sobre el comportamiento de su target.

El Search Engine Optimization es una disciplina que, al igual que cualquiera del ámbito digital, se actualiza constantemente, lo que implica un perfil de profesional SEO en continua formación y un futuro del SEO incierto e incapaz de predecir. Como ad- 
vierte Barquilla en la entrevista realizada para este trabajo: "No sé cómo será el futuro del SEO pero sí sé que es un factor que cada vez más depende de un tercero, llámese Google o llámese futuro buscador de uso mayoritario..."

\section{Conclusiones y discusión:}

Decía Kapuscinsky (2004) que son cinco los sentidos que ha de desarrollar un buen periodista: "estar, ver, oír, compartir y pensar". También advertía que para ser un periodista había que "ser buena persona", que no es oficio hecho para los cínicos (Kapuscinsky, 2006). Las reflexiones del cronista polaco podrían parecer obviedades anacrónicas en el ecosistema digital del tercer milenio pero no lo son. Ni siquiera cuando afrontamos desarrollos tan incipientes y relacionados con el impacto de la tecnología y el poder de los algoritmos que centra este trabajo.

Kapuscinsky estaba pensando en el profesional tradicional, el de raza, el del olfato y los principios éticos, el de unos medios que aún ni vislumbraban la convergencia tecnológica que haría tambalear el sistema en los años 90. Medio siglo después, la esencia en el ejercicio del periodismo se mantiene. Por encima de necesidades imperiosas de adaptación, de reciclaje continuo y de aprendizaje a contrarreloj.

Pero el mundo digital ha traído consigo un aluvión de cambios que, como hemos visto en este trabajo, está transformando también el ejercicio del oficio. El nuevo paradigma de comunicación, con la irrupción de los algoritmos como activadores y condicionantes de todo el proceso, tiene también una repercusión dentro de las redacciones incorporando nuevos perfiles pero imponiendo también la implantación de toda una cultura SEO de forma transversal en las distintas áreas y secciones. Redactar un buen contenido, atractivo y orientado al usuario, tener creatividad y visión estratégica o aprovechar al máximo el altavoz que suponen las redes sociales son factores que resul- tan fundamentales a la hora de elaborar un buen contenido SEO, sin olvidar la importancia que conlleva tener ciertos conocimientos en técnicas y manejo de gestores de contenidos.

Desde esta perspectiva, y partiendo de la oportunidad que ha significado poder analizar e investigar desde dentro el proyecto de desarrollo SEO en un medio como Diario de Sevilla, creemos que este trabajo no solo da respuesta a los objetivos planteados en cuanto a identificación de perfil, técnicas y estrategias, sino que también se presenta a modo de manual de uso que cualquier periodista o redacción podría aplicar para implementar el SEO en la producción de noticias y como parte de la cultura de funcionamiento del propio cibermedio.

Estamos en un nuevo ecosistema en el que se estrecha la relación entre el SEO y el periodismo y en un doble sentido: responder de forma más precisa y acertada a las necesidades de información de su audiencia y conseguir la supervivencia misma de las empresas alcanzando unos buenos niveles de tráfico de usuarios que garanticen unos ingresos publicitarios y su propia viabilidad.

Hay, no obstante, una incertidumbre y una pregunta incómoda que se desliza en estos planteamientos: si el SEO ayudará al Periodismo a reconciliarse con las audiencias, a hacer mejor periodismo, o todo lo contrario. Si el impacto de los algoritmos podrá canalizarse dentro de las redacciones para optimizar al máximo las posibilidades que da la tecnología o terminará prostituyendo y desvirtuando el oficio. Nos enfrentamos, de nuevo, a las complejas tensiones que han marcado la historia misma del Periodismo. Con una respuesta que está por escribir. De entrada, resulta paradójico que, justo cuando los medios ven tambalearse su papel de intermediarios, sean justamente los algoritmos lo que tomen su función. Un papel que se presenta más necesario que nunca en el contexto de desinformación y saturación que ha impuesto el tablero digital.

\section{Referencias bibliográficas}

Amoedo, A. (2021). El acceso algorítmico por redes, búsquedas de tema y agregadores (57\%) mantiene su ventaja sobre las marcas de medios (50\%). Digital News Report España 2021. https://bit.ly/3fsyAR9.

Angrosino, M. (2012). Etnografía y observación participante en Investigación Cualitativa. Colección Investigación Cualitativa. Editorial Morata.

Aruguete, N. (2009). Estableciendo la agenda. Los orígenes y la evolución de la teoría de la Agenda Setting. Universidad Católica Argentina. Instituto de Comunicación Social, Periodismo y Publicidad. https://bit.ly/3f8ZJbE.

Brightedge (2018). Organic Search Is Still the Largest Channel. https://bit.ly/3ybM7Us

Booth, A., Papaionnou, D. y Sutton, A. (2012). Systematic Approaches to a Successful Literature Review. Sage.

Cabezuelo Lorenzo, F. y Rodríguez Escanciano, I. (2007). Del periodismo 2.0 al 3.0. blogs y otras nuevas formas de participación de los públicos. En La ética y el derecho de la información en los tiempos del postperiodismo (pp. 207-222). Fundación COSO. https://bit.ly/3rENrwu.

Caldevilla Domínguez, D. (2010). Las Redes Sociales. Tipología, uso y consumo de las redes 2.0 en la sociedad digital actual. Documentación de Ciencias de la Información, 33, 45-68.

Castillo, C. del (2021). La alemana GfK sustituye a Comscore en la medición de audiencias digitales en España en un período clave para el sector. elDiario.es. https://bit.ly/3iY3MZj 
Codina, L. (2020). Revisiones bibliográficas sistematizadas en Ciencias Humanas y Sociales. 1: Fundamentos. En C. Lopezosa, J. Díaz-Noci, L. Codina (Eds), Anuario de Métodos de Investigación en Comunicación Social, 1. Universitat Pompeu Fabra. https://repositori.upf.edu/handle/10230/44601.

Curiel, E. H. (2011). El periodismo en el siglo de las redes sociales. Vivat Academia (117E), 1113-1128. https://doi. org/10.15178/va.2011.117E.1113-1128.

Dod, R. (2019). How Social Media Helps SEO [Final Answer]. Search Engine Journal. https://bit.ly/37549f2.

Durán Pila, G. y Caldera Serrano, J. (2014). Presencia de la información audiovisual en los periódicos digitales españoles. Documentación de las Ciencias de la Información, 37, 221-229.

Giomelakis, D., Karypidou, C. y Veglis, A. (2019). SEO inside Newsrooms: Reports from the Field. Future Internet 2019, $11,261$.

Hart, C. (2008). Doing a Literature Review: Releasing the Social Science Research Imagination. Sage.

Iglesias-García, M. y Codina, L. (2016). Los cibermedios y la importancia estratégica del posicionamiento en buscadores (SEO). Opción: Revista de Ciencias Humanas y Sociales, 9, 929-944. https://bit.ly/3xcJtMH.

Kapuscinski, R. (2004). Los cinco sentidos del periodista: estar, ver, oir, compartir, pensar. Fondo de Cultura Económica.

Kapuscinski, R. (2006). Los cínicos no sirven para este oficio (Sobre el buen periodismo). Anagrama.

Lopezosa, C., Codina, L., Díaz-Nocy, J. y Ontalba-Ruipérez, J. A. (2020). SEO y cibermedios: de la empresa a las aulas. Revista Comunicar, 63(XXVIII), 65-75. https://doi.org/10.3916/C63-2020-06.

Lopezosa, C., Codina, L. y Pérez-Montoro, M. (2019). SEO and Digital News Media: Visibility of Cultural Information in Spain's Leading Newspapers. Tripodos, 44, 41-61.

Lopezosa, C., Trillo-Domínguez, M., Codina, L., y Cabrera Méndez, M. (2021). El SEO en la empresa periodística: percepciones y elementos clave para su adopción en las redacciones. Revista Latina De Comunicación Social, 79, 27-45. https://doi.org/10.4185/RLCS-2020-1487.

Luna, A. C. (2017). Posicionamiento web (SEO/SEM). ICB editores.

McCombs, M. y Shaw, DL. (1972). La función de establecimiento de la agenda de los medios de comunicación. Opinión pública trimestral, 36(2), 176-187. https://bit.ly/3ibm5ef.

Murga, G. (2017). Amazon Takes 49 Percent of Consumers' First Product Search. But Search Engines Rebound. https:// bit.ly/3BT9swj.

Orihuela, J. L. (2004). eCommunication: the 10 paradigms of media in the digital age. En R. Salaverría y Ch. Sádaba (Eds.), Towards new media paradigms: Content, producers, organizations and audiences (pp. 129-136). Ediciones Eunate. https://bit.ly/3fbObEu.

Purcell, K. (2011). Search and Email Still Top the List of Most Popular Online Activities. Pew Research Center. https:// pewrsr.ch/3rHrop0.

Salaverría, R. (2005). Cibermedios. El impacto de internet en los medios de comunicación e España. Comunicación Social.

Sánchez-Guijaldo, M. P. (2017). Periodismo ciudadano, ¿un nuevo fenómeno de periodismo? Documentación de las Ciencias de la Información, 40, 31-54. http://dx.doi.org/10.5209/DCIN.57161.

Sentance, R. (2016). The Continuing Rise of Voice Search and How You Can Adapt to It. Search Engine Watch. https:// bit.ly/2TFzYrG

Trillo-Domínguez, M. (2015): La prensa andaluza: cuando despertamos de la crisis, el dinosaurio todavía estaba allí. En F. J. Gómez Pérez (Coord.), Politicas de impulso a las industrias audiovisuales (pp. 47-64). Comunicación Social Ediciones.

Trillo-Domínguez, M. y Alberich-Pascual, J. (2020). Análisis y tipificación de formatos emergentes en el ciberperiodismo español: de la adaptación multimedia a la disrupción transmedia. Estudios sobre el Mensaje periodístico, 26(1), 367375. https://dx.doi.org/10.5209/esmp.67317.

Varela, J. (2005). Blogs vs. MSM. Periodismo 3.0, la socialización de la información. Telos: Cuadernos de comunicación e innovación, $65,68-76$. 